\title{
Post-traumatic Stress Disorder and Post-traumatic Growth in Breast Cancer Patients - a Systematic Review
}

\author{
Darshit Parikh ${ }^{1}$, Paolo De Ieso ${ }^{2}$, Gail Garvey ${ }^{3}$, Thanuja Thachil ${ }^{2}$, Ramya \\ Ramamoorthi $^{1}$, Michael Penniment ${ }^{2}$, Rama Jayaraj ${ }^{1 *}$
}

\begin{abstract}
Breast cancer $(\mathrm{BC})$ is potentially a traumatic stressor which may be associated with negative outcomes, such as post-traumatic stress disorder (PTSD) or positive changes, such as post-traumatic growth (PTG). This study aims to identify the core issues of BC related PTSD, PTG and psychological distress by interrogating the literature in BC survivors. We have also highlighted issues related to the assessment, diagnosis and clinical management of PTSD and PTG. The authors systematically reviewed studies published from 1985 to 2014 pertaining to PTSD, psychological distress and PTG in BC survivors with particular attention paid to incidence rates and causative factors. Multiple studies intimated that women with BC have evidence of PTSD at the initial stages of diagnosis, whereas PTG develops once patients undergo treatment. Early diagnosis and treatment of PTSD/PTG is paramount from literature review but the previously mentioned relationship between PTSD and PTG in BC patients could not be verified. It is evident from the literature that a small percentage of BC patients experience PTSD, while the majority experience PTG after BC diagnosis and treatment. Future research should include prospective studies focusing on high-risk patients, causative factors and the development of psychological interventions.
\end{abstract}

Keywords: Breast cancer - PTSD - PTG - psychological status - quality of life - psychological distress

Asian Pac J Cancer Prev, 16 (2), 641-646

\section{Introduction}

Breast cancer (BC) is one of the most common cancers affecting women world-wide and is increasing in developing countries due to increasing life expectancy, urbanisation and adoption of the western lifestyle (Niu et al., 2014; World Health Organisation, 2014a). In 2011, it was estimated that 508,000 women died globally from BC (Tedeschi and Calhoun, 2004; Rustad et al., 2012; World Health Organisation, 2014a). BC is also a problem in the developed world, accounting for $50 \%$ of all world-wide BC diagnoses and up to $58 \%$ of all deaths from BC (World Health Organisation, 2014b). In most developing countries, $\mathrm{BC}$ incidence rates are below $40 \%$ (World Health Organisation, 2014b). BC incidence rates differ world-wide with rates in North America, Sweden and Japan around 80\%,60\% in middle-income countries and below $40 \%$ in low-income countries (World Health Organisation, 2014b).

The number of new BC diagnoses in Australia increased from 5,303 in 1982 to 14,181 in 2010. In 2014, approximately 15,270 Australian women are expected to be diagnosed with $\mathrm{BC}$, and estimated to increase to 17,210 by 2020 (Cancer Australia, 2014). BC survival rates have improved in recent years in developed countries with survival rates in Australia of approximately $89 \%$ between 2006-2010 (Cancer Australia, 2014). The improved survival rates are attributed in part to early diagnosis (World Health Organisation, 2014b), treatment affordability (Coleman et al., 2008) and timely access to crucial treatment modalities (World Health Organisation, 2014b).

Common symptoms after BC diagnosis include fatigue, sexual problems, cognitive dysfunction and a perceived fear of recurrence (Chan et al., 2011; Abu-Helalah et al., 2014). Previous studies noted that the majority of women with BC exhibited high levels of distress, appearance and body image concerns and psychological dysfunction in the first year after BC treatment (Tjemsland et al., 1998; Shelby et al., 2008; Ho et al., 2011; Abu-Helalah et al., 2014). BC survivors gradually appear to adapt to life with the ever present concerns of disease recurrence and the subsequent development of positive stress, known as PTG (Tedeschi and Calhoun, 2004). This manifests in BC survivors as increased confidence, engagement in spiritual practices, family engagement and practices which enhance positive social interactions (Lelorain et al., 2010). Conversely, PTSD reduces cancer survivor's confidence

${ }^{1}$ School of Psychological and Clinical Sciences, Charles Darwin University, ${ }^{2}$ Northern Territory Radiation Oncology, Alan Walker Cancer Care Centre, Darwin, Northern Territory, ${ }^{3}$ Division leader of Epidemiology and Health Systems, Menzies School of Health Research, Adelaide Street, Brisbane, Australia*For correspondence: Rama.Jayaraj@cdu.edu.au 
and increases fear of disease recurrence and death (Kangas et al., 2002). Many studies have investigated both PTSD and PTG, but there is a paucity of data comparing the frequency, causative factors and effects of PTSD and PTG on BC survivors (Tjemsland et al., 1998; Cordova et al., 2001; Ho et al., 2011; Koutrouli et al., 2012). As a consequence, further targeted research should be strongly considered in this area (Bellizzi and Blank, 2006; Arpawong et al., 2013; Arving et al., 2013; ).

This paper aims to interrogate the literature regarding the positive impact of PTG, and negative impact of PTSD and psychological distress in BC survivors. It also compares different aspects of post-diagnostic psychological challenges, including psychological tools in use and treatment choices.

\section{Materials and Methods}

Literature search: Peer-reviewed research articles regarding $\mathrm{BC}$ and $\mathrm{PTSD}$, psychological distress and

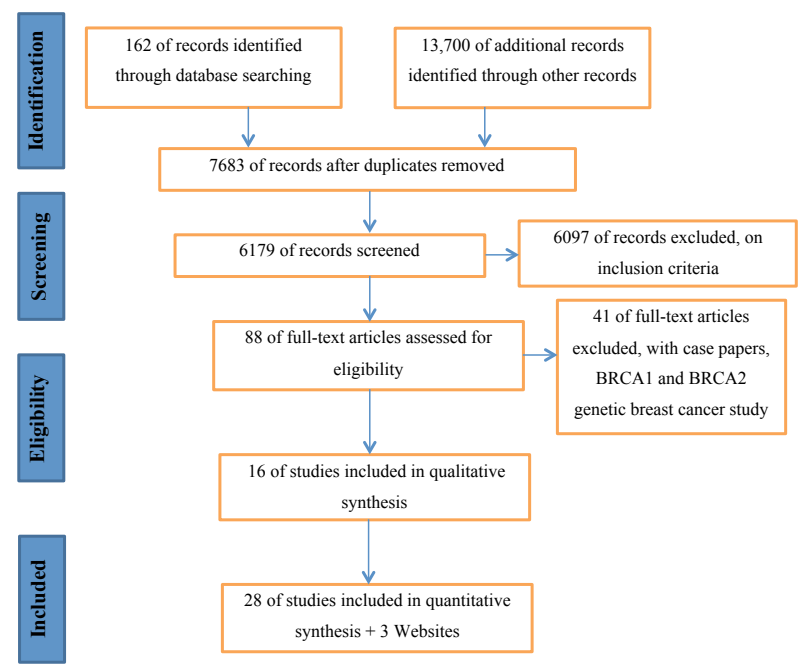

Figure 1. Flow of information through the Different Phases of a Systematic Review. Figure is based on PRISMA statement: Moher et al., 2009 [43]
PTG were identified through searches in the following electronic databases: PubMed, MEDLINE, Science Direct, Google Scholar, Cochrane library and published materials including those published in Australian health reports, American health database and websites. After a series of previous search attempts with other words in the databases, the most suitable combinations of key words relating to the proposed objectives were: BC and PTSD, psychological distress, PTG, PTG and PTSD in BC survivors. The selected abstracts were assessed by a study team to verify whether their content was accurate and appropriate for inclusion.

Inclusion criteria: $i$ ) Papers published from 1985-2014 in the scientific literature, focussing on BC, effects of PTSD, PTG and psychological distress after diagnosis and/or treatment. ii) Only articles published in English were considered.

Exclusion criteria: iii) Articles exclusively related to $\mathrm{BC}$ associated with BRCA1 and BRCA2 gene mutations.

\section{Results}

Initial database searches using key words explained above yielded 7683 results. When the search was further refined (Figure 1), there were 88 articles found that met inclusion criteria. Abstracts from the 88 articles were retrieved and assessed by the review team, with 47 articles/ government websites included in the final analysis.

The review team found five different types of subjects studied, including: $i$ ) PTSD in BC survivors (Andrykowski et al., 1998; Andrykowski et al., 2000; Amir and Ramati, 2002; Gurevich et al., 2002; Kangas et al., 2002; Levine et al., 2005; Kangas et al., 2007; Knobf, 2007; Mehnert and Koch, 2008; Shelby et al., 2008; Smith et al., 2008; Bellizzi et al., 2010; Nordin et al., 2012; Rustad et al., 2012; Arving et al., 2013; Perez et al., 2014).

ii) PTG in BC survivors (Tedeschi and Calhoun, 1996; Sears et al., 2003; Tedeschi and Calhoun, 2004; Thewes et al., 2004; Bellizzi and Blank, 2006; Lelorain et al., 2010; Arpawong et al., 2013; Brix et al., 2013; Danhauer et al., 2013).

Table 1. Studies Investigating on PTSD and its Effects on Breast Cancer Patient Samples

\begin{tabular}{|c|c|c|c|c|}
\hline$\overline{\text { Studies }}$ & Study Design & Sample Size & Measure & Time \\
\hline Andrykowski et al., 1998 & Cross-sectional & 82 & PCL-C & Mean of 37 months post treatment \\
\hline Andrykowski et al., 2000 & $\begin{array}{l}\text { Cross-sectional } \\
\text { and longitudinal }\end{array}$ & 46 & $\begin{array}{l}\text { PCL-C and interview } \\
\text { at follow up }\end{array}$ & $\begin{array}{l}\text { Mean } 29.8 \text { months post treatment, } \\
1 \text { year follow up }\end{array}$ \\
\hline $\begin{array}{l}\text { Mehnert and Koch., } 2008 \\
\text { and longitudinal }\end{array}$ & Cross-sectional & 1083 & PCL-C & 47 months after diagnosis \\
\hline Shelby et al., 2008 & Cross-sectional & 74 & PCL-C, SCID & $30-40$ months post diagnosis \\
\hline Kangas et al., 2007 & Longitudinal & 82 & DSM-IV & Post diagnostic \\
\hline Perez et al., 2014 & $\begin{array}{l}\text { Cross-sectional } \\
\text { and Longitudinal }\end{array}$ & 187 & $\begin{array}{l}\text { Stanford Acute Stress } \\
\text { Reaction Questionnaire }\end{array}$ & Post diagnostic 6 to 12 months \\
\hline Bellizzi et al., 2010 & Multivariate analysis & 802 & PTGI & Median age 52.7 , post diagnostic \\
\hline Arving et al., 2013 & $\mathrm{RCT}$ & 300 & Psychological intervention & Age more then 18 \\
\hline Nordin et. Al., 2012 & RCT & 300 & IES & Age more then 18 \\
\hline Smith et al. 2008 & $\mathrm{RCT}$ & 886 & $\begin{array}{l}\text { Self-Administered } \\
\text { Comorbidity Questionnaire }\end{array}$ & 2 years post diagnostic \\
\hline Levine et al., 2005 & $\mathrm{RCT}$ & 181 & PCL-C & 18 months \\
\hline Amir and Ramati., 2002 & Cross-sectional & 78 & DSM-III-r, scl-90, whoqol-Brief & 5 years post diagnosis \\
\hline
\end{tabular}

*RCT: Randomised Control Trial; PCL-C: Posttraumatic stress disorder checklist-Civilian Version; IES: Impact of Event Scale; DSM-IV: Diagnostic and Statistical manual of Mental Disorders, Fourth edition; WHOQOL-BRIEF: World Health Organisation Quality of Life- Brief questionnaire 
Table 2. Studies Investigating on Psychological Distress and its Effects on Breast Cancer Patients

\begin{tabular}{llcll}
\hline Studies & Study Design & Sample Size & \multicolumn{1}{c}{ Measure } & Time \\
\hline Abu-Helalah et al., 2014 & Cross-sectional & 236 & EORTC QLQ-C30, HADS & 1-3 years after diagnosis \\
Arpawong et al., 2013 & Cross-sectional & 114 & PTGI & N/A \\
Bellizzi \& Blank, 2006 & Cross-sectional & 215 & PTGI & 1-4 years after treatment \\
Brix. et al., 2013 & Data analysis & 1645 & PTGI & 18 years \\
Chang et al., 2014 & Cross-sectional & 126 & EORTC QLQ-C30 & 12 months after diagnosis \\
Rizalar et al., 2014 & Cross-sectional & 100 & PAIS-SR & After six months of diagnosis \\
Sears et al., 2003 & Longitudinal study & 92 & PTGI and qualitative interview & 6 to 18 month post-surgery \\
Weiss et al. 2004 & Cross-sectional & 72 couples & PTGI & 39 months post diagnosis \\
Leorain et al., 2010 & Cross-sectional & 307 & PTGI & 5 years post treatment \\
Danhauer et al., 2013 & Cross-sectional & 653 & PTGI & Within 8 months of diagnosis, \\
& & & & then 6,12, 18 months \\
Yavuzsen et al., 2014 & Pilot Study & 16 & Group Therapy Program & After treatment \\
\hline
\end{tabular}

*PTGI: Posttraumatic Growth Inventory, EORTC QLQ-C30 : The European Organisation for Research and Treatment of Cancer Quality of life Questionnaire, HADS : The Hospital Anxiety and Depression Scale, PAIS-SR : Psychological Adjustment to Illness Scale-Self-reflection

Table 3. Studies Focusing on Assessment of Both PTSD and PTG and its Effects on Breast Cancer Patients

\begin{tabular}{|c|c|c|c|c|}
\hline Studies & Study Design & Sample Size & Measure & Time \\
\hline Cordova et al., 2001 & Cross-sectional & 70 & PTGI & 2 years post diagnosis \\
\hline Ho et al., 2011 & Cross-sectional & 83 & CIES-R & Post diagnostic \\
\hline Tjemsland et al., 1998 & Longitudinal Study & 106 & IES and GHQ-28 & $\begin{array}{l}\text { day before surgery, } \\
\text { after one year of surgery }\end{array}$ \\
\hline Chan et al., 2011 & Cross-sectional & 170 & $\begin{array}{l}\text { The Chinese version of attention to } \\
\text { positive and negative } \\
\text { information scale (CAPNIS) }\end{array}$ & Within a year of diagnosis \\
\hline Liu et al., 2014 & Longitudinal Study & 120 & PTGI and Distress screening measures & $\begin{array}{l}3 \text { months post diagnosis } \\
\text { then } 6 \text { and } 9 \text { months }\end{array}$ \\
\hline
\end{tabular}

*CIES-R: The Chinese Impact of Event Scale- Revised; IES: Impact of Event Scale; GHQ-28: General Health Questionnaire- 28 Items

Table 4. Studies Discussing Literature Reviews on PTSD, PTG and PTSD and PTG Together

\begin{tabular}{ll}
\hline PTSD Review & Study Design \\
\hline Rustad et al., 2012 & Review article \\
Kangas et al., 2002 & $\begin{array}{l}\text { Conception and } \\
\text { empirical review } \\
\text { review report }\end{array}$ \\
Gurevich et al., 2002 & Review study \\
Knobf., 2007 & Study Design \\
PTG review & Review article \\
Tedeschi and Calhoun, 2004 & Review article, PTGI study \\
Tedeschi \& Calhoun., 1996 & Study Design \\
PTSD \& PTG review & Systematic Review \\
Koutrouli et al., 2012 & Review \\
Sumalla et al., 2009 & Perspective review \\
Jones., 2001 &
\end{tabular}

iii) Both PTSD and PTG in BC survivors (Tjemsland et al., 1998; Cordova et al., 2001; Jones, 2001; Sumalla et al., 2009; Ho et al., 2011; Chan et al., 2011; Koutrouli et al., 2012; Liu et al., 2014). iv) PTSD and psychological distress and diagnostic challenges in the same patient population (Gurevich et al., 2002; Tedeschi and Calhoun, 2004; Bellizzi et al., 2010; Rustad et al., 2012; Yavuzsen et al., 2012; Abu-Helalah et al., 2014; Chang et al., 2014; Rizalar et al., 2014) and v) Treatment approaches (Andrykowski et al., 2000; Levine et al., 2005; Knobf, 2007; Bellizzi et al., 2010; Assi et al., 2013; Liu et al., 2014)

\section{Discussion}

PTSD is a psychiatric condition that occurs as a result of exposure to a traumatic event, defined as a threat of death or serious injury (Kangas et al., 2002). This in turn evokes intense fear, helplessness, or horror (DSMIV, 2000). Previous studies revealed that nearly $41 \%$ of women diagnosed with $\mathrm{BC}$ had a high level of distress and $11 \%$ of women had major depressive disorders (Chan et al., 2011). Prevalence rates of BC related PTSD varied from 3 to $19 \%$ in the literature (Andrykowski et al., 2000). There are multifactorial causes/risk factors for PTSD such as cancer stage, prognosis, patient-specific treatment approaches, presence or absence of pain, performance status, need for hospitalization and degree of social support (Kangas et al., 2002; Niu et al., 2014; Rizalar et al., 2014). After diagnosis, a BC patient's worries appear to increase gradually as concerns increase regarding treatability of the disease and prognosis (Deimling et al., 2006). The incidence of PTSD in BC patients ranged from $1 \%$ to $58 \%$ in one paper (Kangas et al., 2002), while other studies were more specific, with a PTSD rate of more than 20\% (Cordova et al., 2001; Chan et al., 2011). Moderate to high levels of fear associated with concerns of disease recurrence were noted in $56 \%$ of Dutch BC survivors (Deimling et al., 2006). Other studies have focussed on particular predictive factors for PTSD, including effects of negative stressors like young age at diagnosis (Andrykowski et al., 2000; Andrykowski et al., 1998), lower socio economic status (Cordova et al., 2001), low educational achievement (Cordova et al., 2001; Chang et al., 2014), emotional social environment (Tjemsland et al., 1998), poor social support and reduced physical activity (Smith et al., 2008). The above mentioned factors are commonly predictive of PTSD after BC diagnosis. Many studies have highlighted that the effects and psychological 
symptoms of cancer treatment could be long lasting and widespread (Andrykowski et al., 2000; Amir and Ramati, 2002). As a consequence, early diagnosis and timely psychological intervention is necessary for those at risk of PTSD and could help to curtail symptoms (Amir and Ramati, 2002; Smith et al., 2008; Perez et al., 2014).

It is clear that $\mathrm{BC}$ diagnosis is a trigger for the development of numerous traumatic psychological reactions for the patient (Kangas et al., 2002). Patients who face life threatening diseases like $\mathrm{BC}$ experience different stressful events including financial and social worries, fear of recurrence, psycho-social pressure from family and fear of death (Nordin et al., 2012; Arving et al., 2013; Rizalar et al., 2014). The Diagnostic and Statistical Manual of Mental Disorders, Fourth Edition (DSM-IV) report categorises these symptoms as phobias and anxiety (DSM-IV, 2000).

Psychological distress happens at different stages of the cancer journey. Mullan described three stages of cancer survival, as patient traverse the $\mathrm{BC}$ treatment pathway; $i$ ) The first acute phase involves the institution of aggressive treatment, when the patient faces overwhelming emotional stress and fear of potential death; ii) The second stage begins when formal treatment finishes and disease recurrence is at the forefront of the patient's thoughts. iii) The third phase is described as a permanent survival phase, and is comparatively less stressful as fear of recurrence becomes less likely (Mullan, 1985).

The process of coping with a chronic stressor such as BC creates long term psychological distress (Bellizzi et al., 2010). Bellizzi argues that the perceived ongoing threat of $\mathrm{BC}$ recurrence leads to long term stress and can develop into chronic psychological conditions (Bellizzi et al., 2010). Chang and colleagues in their research found that altered appearance such as loss or disfigurement of breasts, discoloured skin, and hair loss results in psychological distress and affects their quality of life (Chang et al., 2014). Another report indicated that psychosocial distress experienced within the first year post diagnosis has a huge impact on psychosocial wellbeing and quality of life in long term BC survivors (Mehnert and Koch, 2008). Andreu and colleagues revealed in a study of 102 BC survivors that psychological factors such as emotional suppression, strategies for coping with treatment and gaining social support from community and family were conducive to improved psychological status (Andreu et al., 2012). Thewes and colleagues investigated psychological distress in pre and post-menopausal women. It was found that the majority of women in both groups could cope with the trauma of $\mathrm{BC}$ diagnosis and treatment. Some patients needed psychological support, a finding more often noticed in the premenopausal subgroup (Thewes et al., 2004). The authors concluded that premenopausal women were more concerned about recurrence, experienced more painful memories of treatment, had more detrimental effects to their careers and social status than their postmenopausal counterparts (Thewes et al., 2004). Jones highlighted that as physical symptoms of disease and treatment reduce, symptoms of psychological distress would resolve over a period of time in the majority of women (Jones, 2001).

In some BC survivors, persistent emotional distress can lead to psychological pathology, including anxiety disorders or clinical depression (Nordin et al., 2012). Approximately $80 \%$ of patients with $\mathrm{BC}$ reported high levels of stress at diagnosis. These included negative intrusive thoughts and avoidance behaviour towards treatment (Nordin et al., 2012). Studies done using self-report screening tools (e.g., Hospital Anxiety and Depression Scale, PTSD Checklist Civilian Version, and Short-Form Health Survey) have reported distress, anxiety and depression in one-quarter to one-third of women who present with $\mathrm{BC}$, and the incidence of symptoms can increase gradually up to $50 \%$ after diagnosis. (Mehnert and Koch, 2008; Arving et al., 2013). Mehnert and Koch explained that psychological distress was more likely in the first year after diagnosis. There was a steady and gradual improvement in distress, psychological wellbeing and quality of life in long term survivors as the time from diagnosis increased (Mehnert and Koch, 2008).

Post-traumatic growth (PTG) was initially described by Tedeschi and Calhoun (1996). PTG is considered as "positive psychological change experienced as a result of the struggle with highly challenging life circumstances" (Tedeschi and Calhoun, 2004; Brix et al., 2013; Danhauer et al., 2013). PTG in BC is a well described and accepted psychological phenomenon (Cordova et al., 2001; Tedeschi and Calhoun, 2004; Chan et al., 2011). Danhauer and colleagues defined PTG as a positive change and psychological transcendence from a challenging life experience (Danhauer et al., 2013). PTG includes the development of hope and optimism after the diagnosis of $\mathrm{BC}$, serving as a protective mechanism against depression, distress and psychological deterioration (Bellizzi and Blank, 2006; Danhauer et al., 2013). Different studies have documented positive life changes in the later phases of life for those who have faced traumatic events like BC (Cordova et al., 2001; Lelorain et al., 2010). Sears and colleagues support this finding as more than $80 \%$ of BC survivors were found to benefit from the psychologically stressful BC experience (Sears et al., 2003). Tedeschi and Calhoun also emphasised that PTG was an emotional evolution to a new personality and its subsequent benefit to psychological stability in the BC survivor (Tedeschi and Calhoun, 2004).

PTG in BC survivors is more likely in those of younger age, longer time since diagnosis, greater cancer related stress, positive/adaptive coping, religious coping, sharing one's BC experience, and for those seeking social support (Danhauer et al., 2013). PTG was more likely to occur in the first year after diagnosis, corresponding to a time when one's assumptions about the world and oneself were most likely to be challenged (Danhauer et al., 2013). Liu and colleagues found 'personal growth', and 'relating to others' as hallmark of PTG development (Liu et al., 2014).

Regarding ethnic differences in PTG, Bellizzi et al highlighted that African-American BC survivors were more likely to experience higher levels of PTG than White and Hispanic women (Bellizzi et al., 2010). PTG has been reported in $87 \%$ of $\mathrm{BC}$ survivors in one series, which highlighted a reduced incidence of PTG in those with lower general levels of health, increased experience of pain, and interference with activities of daily living and 
work (Arpawong et al., 2013).

There are common issues which persist in some women with BC and PTSD such as threat to life and body integrity including disfigurement, disability, pain, and loss of social and occupational roles (Gurevich et al., 2002; Tedeschi and Calhoun, 2004). Yavuzsen and colleagues revealed that supportive care programs that help patients better understand and cope with their symptoms may result in reduced frequency, duration and intensity of treatment related symptoms (Yavuzsen et al., 2012). Gurevich and colleagues explained that long term cancer symptoms could lead to long term psychological anomalies which can develop into PTSD (Gurevich et al., 2002). Limited research has focussed on the identification of PTSD after BC diagnosis. (Sumalla et al., 2009).

The major issue pertinent to PTSD in BC survivors is a well described difficulty to diagnose PTSD at early stages of diagnosis and treatment (Kangas et al., 2007). The reason for this is that the well-established PTSD diagnostic tools are less sensitive at earlier stages of diagnosis (Rustad et al., 2012; Kangas et al., 2007). Diagnosis of BC related PTSD (at all stages) is clinically important. It is also important to develop a cost effective and reliable diagnostic tool for BC related PTSD (Andrykowski et al., 1998).

After reviewing 5 studies that utilised quantitative methods, all relied on the PCL-C diagnostic tool. (Andrykowski et al., 1998; Andrykowski et al., 2000; Levine et al., 2005; Mehnert and Koch, 2008; Shelby et al., 2008) Rustad and colleagues used PTSD Checklist- for Civilians (PCL-C) tool in the diagnosis of PTSD (Rustad et al., 2012). The author also discussed trauma-focused cognitive behavioural therapy for cancer patients, a technique which can also be implemented for women with BC (Rustad et al., 2012). The Post-traumatic Stress Symptomology (PTSS) tool is a diagnostic apparatus which can be used to evaluate the degree of symptoms after diagnosis of PTSD, as post-traumatic stress symptoms fluctuate over a period of time from BC diagnosis and treatment (Perez et al., 2014). Andrykowski et al performed a cross-sectional and longitudinal study investigating PTSD with the PCL-C tool. It was found that the false positive rate was unacceptably high in these studies but they were unable to find an accurate diagnostic tools to measure PTSD in patients with BC (Andrykowski et al., 2000).

The post-traumatic growth inventory (PTGI) is a commonly utilised tool to measure PTG and provides separate scores in five domains of life such as relationships with other, new possibilities, appreciation of life, spirituality, and personal strength (Bellizzi et al., 2010).

Effective and accurate diagnosis with timely psychological treatment approaches could help to reduce psychological distress (Levine et al., 2005). Levine and colleagues found that the prevalence rate of PTSD symptoms was approximately $14 \%$ after BC diagnosis and that early psychological interventions effectively reduced PTSD and psychological distress (Levine et al., 2005). Andrykowski and colleagues argued that symptoms of PTSD did not improve over time with psychological interventions in women with BC (Andrykowski et al.,
2000), while Knobf highlighted that early detection and intervention can improve women's mental health and psychosocial adjustment pattern among BC survivors (Knobf, 2007).

Feedback from women who had psychological interventions such as PTG inventory and distress management screening were described by Liu and colleagues. They spoke of a perceived benefit to their physical and psychological health outcomes. There was also found to be a weak relationship between PTG and psychological distress, with an increased PTG and decreased psychological distress measured after 6 months of follow-up (Liu et al., 2014). The authors concluded that timely and robust psychological intervention should occur to reduce psychological distress and promote PTG after the diagnosis of BC (Knobf, 2007).

In conclusion, BC survivors face many emotional difficulties and psychological insults after diagnosis. Interventions over the last 15 years have concentrated on improving psychological wellbeing of women with BC. All of these interventions aimed to improve psycho-social involvement, psychological understanding, religious involvement, positive mental changes and reducing effects of PTSD in BC patients. Several experiences used different screening tools and methods to diagnose and measure symptoms of PTSD. The psychological status of women after diagnosis of $\mathrm{BC}$ is often fluctuating, making accurate psychological diagnosis difficult. This is evident in the diagnostic challenge at the initial stage of PTSD. It is clear from the literature that PTG is more common than PTSD in $\mathrm{BC}$ survivors. Findings from relevant studies highlighted that PTG is associated with better life style, happiness and psychosocial wellbeing. Methodological limitations in several studies were evident due to the multifactorial causes of PTSD and PTG. Future research should focus on identifying factors influencing development of PTSD and PTG in BC survivors and their accurate and timely diagnosis/treatment.

\section{References}

Abu-Helalah M, Al-Hanaqta M, Alshraideh H, et al (2014). Quality of life and psychological well-being of breast cancer survivors in Jordan. Asian Pac J Cancer Prev, 15, 5927-36.

Amir M and Ramati A. (2002). Post-traumatic symptoms, emotional distress and quality of life in long-term survivors of breast cancer, a preliminary research. J Anxiety Disord, 16, 195-206.

Andreu Y, Galdon MJ, Dura E, et al (2012). A longitudinal study of psychosocial distress in breast cancer, Prevalence and risk factors. Psychol Health, 27, 72-87.

Andrykowski MA, Cordova MJ, McGrath PC, et al (2000). Stability and change in posttraumatic stress disorder symptoms following breast cancer treatment, a 1-year follow-up. Psychooncol, 9, 69-78.

Andrykowski MA, Cordova MJ, Studts JL, et al (1998). Posttraumatic stress disorder after treatment for breast cancer, Prevalence of diagnosis and use of the PTSD Checklist-Civilian Version (PCL-C) as a screening instrument. J Consul Clin Psy, 66, 586-90.

Arpawong TE, Richeimer SH, Weinstein F, et al (2013). Posttraumatic growth, quality of life, and treatment symptoms among cancer chemotherapy outpatients. Health 
Psychology, 32, 397-408.

Arving C, Thormodsen I, Brekke G, et al (2013). Early rehabilitation of cancer patients - a randomized controlled intervention study. BMC Cancer, 13, 9 .

Assi HA, Khoury KE, Dbouk H, et al (2013). Epidemiology and prognosis of breast cancer in young women. $J$ Thorac Dis, 5, 2-8.

Bellizzi KM, Blank TO (2006). Predicting posttraumatic growth in breast cancer survivors. Health Psychology, 25, 47-56.

Bellizzi KM, Smith AW, Reeve BB, et al (2010). Posttraumatic growth and health-related quality of life in a racially diverse cohort of breast cancer survivors. J Health Psychol, 15, 615-26.

Brix SA, Bidstrup PE, Christensen J, et al (2013). Post-traumatic growth among elderly women with breast cancer compared to breast cancer-free women. Acta Oncol, 52, 345-54.

Cancer Australia (2014). Breast Cancer Statistics. Available at, http://canceraustralia.gov.au/affected-cancer/cancer-types/ breast-cancer/breast-cancer-statistics.

Chan MWC, Ho SMY, Tedeschi RG, et al (2011). The valence of attentional bias and cancer-related rumination in posttraumatic stress and posttraumatic growth among women with breast cancer. Psycho-Oncol, 20, 544-52.

Chang O, Choi EK, Kim IR, et al (2014). Association between socioeconomic status and altered appearance distress, body image, and quality of life among breast cancer patients. Asian Pac J Cancer Prev, 15, 8607-12.

Coleman MP, Quaresma M, Berrino F, et al (2008). Cancer survival in five continents, a worldwide population-based study (CONCORD). Lancet Oncology, 9, 730-56.

Cordova MJ, Cunningham LL, Carlson CR, et al (2001). Posttraumatic growth following breast cancer, a controlled comparison study. Health Psychol, 20, 176-85.

Danhauer SC, Case LD, Tedeschi R, et al (2013). Predictors of posttraumatic growth in women with breast cancer. PsychoOncol, 22, 2676-83.

Deimling GT, Bowman KF, Sterns S, et al (2006). Cancer-related health worries and psychological distress among older adult, long-term cancer survivors. Psychooncol, 15, 306-20.

DSM-IV APATFo. (2000). Diagnostic manual of mental disorders, DSM-IV-TR, Washington, DC, American Psychiatric Association.

Gurevich M, Devins GM, Rodin GM (2002). Stress response syndromes and cancer, conceptual and assessment issues. Psychosomatics, 43, 259-81.

Ho SMY, Chan MWY, Yau TK, et al (2011). Relationships between explanatory style, posttraumatic growth and posttraumatic stress disorder symptoms among Chinese breast cancer patients. Psychology Health, 26, 269-85.

Jones R. (2001). Depression and anxiety in oncology, the oncologist's perspective. J Clin Psychiatry, 62, 52-5.

Kangas M, Henry JL, Bryant RA. (2002). Posttraumatic stress disorder following cancer, A conceptual and empirical review. Clinical Psychology Review, 22, 499-524.

Kangas M, Henry JL, Bryant RA (2007). Correlates of acute stress disorder in cancer patients. J Trauma Stress, 20 , 325-34.

Knobf MT (2007). Psychosocial Responses in Breast Cancer Survivors. Semin Oncol Nurs, 23, 71-83.

Koutrouli N, Anagnostopoulos F, Potamianos G (2012). Posttraumatic stress disorder and posttraumatic growth in breast cancer patients, a systematic review. Women Health, 52, 503-16.

Lelorain S, Bonnaud-Antignac A, Florin A (2010). Long Term Posttraumatic Growth After Breast Cancer, Prevalence, Predictors and Relationships with Psychological Health. $J$ Clin Psychol Med Settings, 17, 14-22.
Levine EG, Eckhardt J, Targ E (2005). Change in post-traumatic stress symptoms following psychosocial treatment for breast cancer. Psychooncol, 14, 618-35.

Liu J-E, Wang H-Y, Wang M-L, et al (2014). Posttraumatic growth and psychological distress in Chinese early-stage breast cancer survivors, a longitudinal study. Psycho-Oncol, 23, 437-43.

Mehnert A and Koch U (2008). Psychological comorbidity and health-related quality of life and its association with awareness, utilization, and need for psychosocial support in a cancer register-based sample of long-term breast cancer survivors. J Psychosom Res, 64, 383-91.

Mullan F (1985). Seasons of Survival, Reflections of a Physician with Cancer. N Engl J Med, 313, 270-73.

Niu HY, Niu CY, Wang JH, et al (2014). Health-related quality of life in women with breast cancer, a literature-based review of psychometric properties of breast cancer-specific measures. Asian Pac J Cancer Prev, 15, 3533-36.

Nordin K, Rissanen R, Ahlgren J, et al (2012). Design of the study, how can health care help female breast cancer patients reduce their stress symptoms? A randomized intervention study with stepped-care. BMC Cancer, 12, 167.

Perez S, Galdon MJ, Andreu Y, et al (2014). Posttraumatic stress symptoms in breast cancer patients, temporal evolution, predictors, and mediation. J Trauma Stress, 27, 224-31.

Rizalar S, Ozbas A, Akyolcu N, et al (2014). Effect of perceived social support on psychosocial adjustment of Turkish patients with breast cancer. Asian Pac J Cancer Prev, 15, 3429-34.

Rustad JK, David D, Currier MB (2012). Cancer and posttraumatic stress disorder, Diagnosis, pathogenesis and treatment considerations. Palliat Support Care, 10, 213-23.

Sears SR, Stanton AL, Danoff-Burg S (2003). The yellow brick road and the emerald city, Benefit finding, positive reappraisal coping and posttraumatic growth in women with early-stage breast cancer. Health Psychol, 22, 487-97.

Shelby RA, Golden-Kreutz DM, Andersen BL (2008). PTSD diagnoses, subsyndromal symptoms, and comorbidities contribute to impairments for breast cancer survivors. $J$ Trauma Stress, 21, 165-72.

Smith SK, Zimmerman S, Williams CS, et al (2008). Posttraumatic stress outcomes in non-Hodgkin's lymphoma survivors. J Clin Oncol, 26, 934-41.

Sumalla EC, Ochoa C, Blanco I (2009). Posttraumatic growth in cancer, Reality or illusion? Clin Psychol Rev, 29, 24-33.

Tedeschi RG and Calhoun LG. (1996). The Posttraumatic Growth Inventory, measuring the positive legacy of trauma. J Trauma Stress, 9, 455-71.

Tedeschi RG, Calhoun LG (2004). Posttraumatic growth, conceptual foundations and empirical evidence. Psychological Inquiry, 15, 1-18.

Thewes B, Butow P, Girgis A, et al (2004). The psychosocial needs of breast cancer survivors; A qualitative study of the shared and unique needs of younger versus older survivors. Psycho-Oncol, 13, 177-89.

Tjemsland L, Soreide JA, Malt UF (1998). Posttraumatic distress symptoms in operable breast cancer III, status one year after surgery. Breast Cancer Res Treat, 47, 141-51.

World Health Organisation (2014a) Breast cancer, Prevention and Control. Available at, http://www.who.int/cancer/ detection/breastcancer/en/.

World Health Organisation (2014b). The global burden of disease. Available at, http://www.who.int/cancer/detection/ breastcancer/en/index1.html.

Yavuzsen T, Karadibak D, Cehreli R, et al (2012). Effect of group therapy on psychological symptoms and quality of life in Turkish patients with breast cancer. Asian Pac J Cancer Prev, 13, 5593-7. 